\title{
Study on Children Voice Features Using Expression Words
}

\author{
HARADA Naoya ${ }^{a}$, NAKATOH Yoshihisa ${ }^{a^{*}}$ \\ ${ }^{a}$ Kyushu Institute of Technology, \\ 1-1 Sensui-cho, Tobata-ku, Kitakyushu-shi, Fukuoka 804-8550, Japan \\ *nakatoh@ecs.kyutech.ac.jp
}

\begin{abstract}
Currently, with the aging of Japan society, the number of hearing impaired people is increasing. Therefore, it can be said that the demand for hearing aids is increasing in Japan. It is said that there are approximately 3.4 million people using hearing aids in Japan. Under these circumstances, $42 \%$ of hearing aid users are discontented, such as difficult to hear the conversation with children. As mentioned above, hearing aid users feel uncomfortable with children voice. We analyzed the characteristics of children voice and then tried to give a processing based on the results to improve the listening satisfies on children speech for hearing aid users. Therefore, we examined expression words which characterizing children voice. Evaluation experiments were carried out using voice of 5 words of 144 boys from a voice database of children. As a result of study, when children changed higher the age, children speech changed from high voice to low voice and from unsteady voice to fluent voice. In addition, from the result of the factor analysis, we found that there are four factors in children speech: "Voice quality and Speaking style", "Voice strength", "Ease of hearing" and "Voice speed". Finally, we compared the evaluation of children's speech given by adults and elderly people respectively.
\end{abstract}

Keywords: hearing aid, children speech.

\section{Introduction}

Currently, Japan is proceeding with aging, and accompanying with it, the number of hearing impaired people is increasing. Therefore, it can be said that the demand for hearing aids is increasing in Japan. It is said that there are approximately 3.4 million people using hearing aids in Japan. Under these circumstances, $42 \%$ of hearing aid users are discontented, such as difficult to hear conversation with children ${ }^{(1)}$. In fact, complaints such as not being able to hear the voice of the child, feeling uncomfortable with the voice of the child echoing can be cited.

As mentioned above, hearing aid users feel uncomfortable with their children speech. We analyzed the characteristics of children speech and tried to process based on the results and improve listening to children speech for hearing aid users. In the present study, we aimed to improve hearing of children speech for hearing aid users. Firstly, we examined expression words that characterize children speech, and decided to analyze what kind of factors are classified.

\section{Children speech database}

In this study, we got a children speech database from the National Institute of Informatics. In the speech database, we used the voice of 5 words of 144 boys aged 6 to 12 . Table 1 shows the specifications of speech database used and used words.

\section{Selection of expression words characterizing children speech}

The expression words used in this research was referred to the previous study on the feature of elderly voice with expression words ${ }^{(2)}$. In the previous study, 11 kinds were selected as expression words of elderly speech, and we decided to use a total of 22 (11 pairs) which combined the negative form of this expression. 


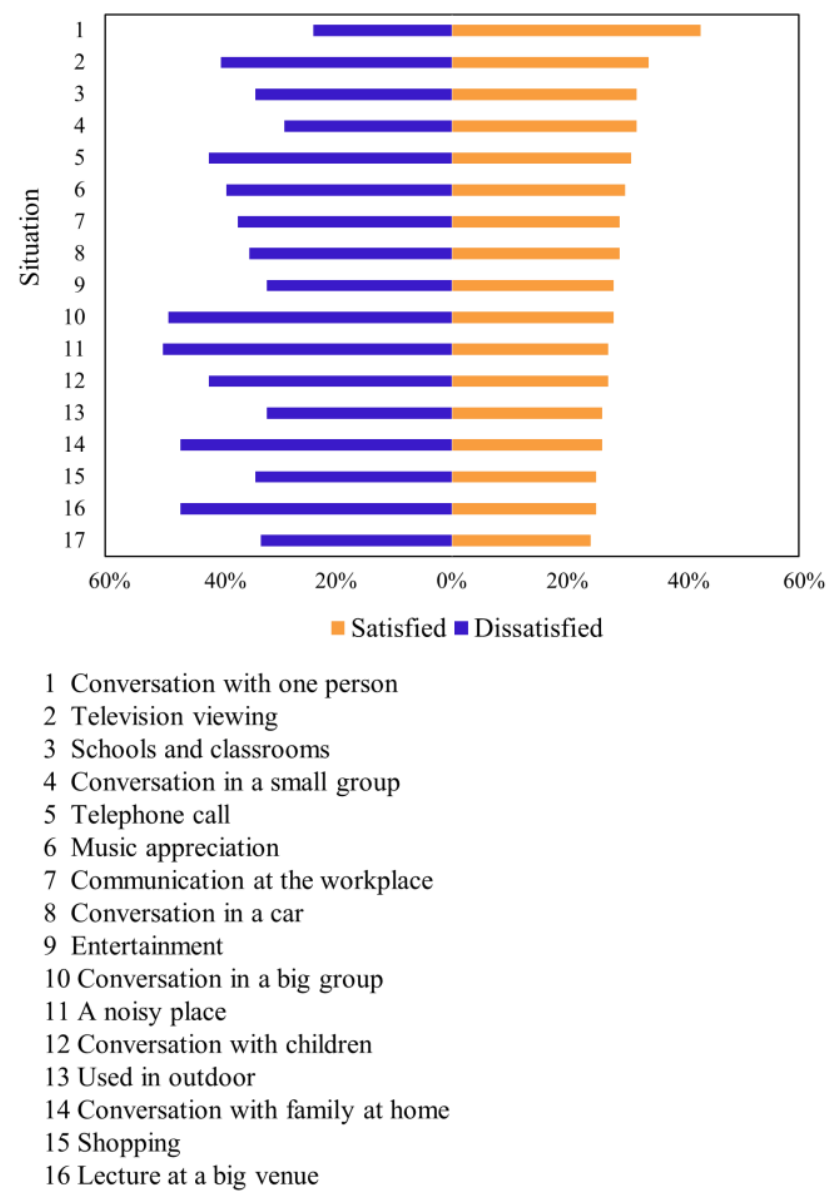

Fig.1 Satisfaction level of hearing aid users

Next, addition of expression words by listening experiment was examined. I asked for 5 adult men to hear the voice of 144 children and asked me to explain how they felt the voice of the children. Two kinds of expression words were added by listening experiments, and a total of 4 (2 pairs) expression words were obtained which combined the negative form of this expression words. Eventually, in this research, we used a total of 26 expressions (13 pairs) expression words combined with expression words selected in previous research and expression words obtained by listening experiments.

\section{Analysis of children speech}

\subsection{The method of experiment}

We evaluated the extent to which the selected expression words can express the actual children speech. We created 144 experimental voices from five words spoken by 144 children. Five adult male subjects were allowed to listen to the experimental voices with headphones and evaluated each of the 13 expression words in five stages of +2 to -2 .
Table 1 Speech database specifications and used words

\begin{tabular}{|c|c|}
\hline & 144 boys \\
& 6 years old 15 people \\
7 years old 25 people \\
Number of people recorded & 9 years old 23 people 29 people \\
& 10 years old 21 people \\
& 11 years old 23 people \\
& 12 years old 8 people \\
\hline Audio file format & wav (16kHz, 16bit) \\
\hline & Calendar \\
& Group \\
Used words & Hyotan \\
& Piman \\
& Peropero-Candy \\
\hline
\end{tabular}

\subsection{Evaluation of expression words characterizing children speech}

The results of the experiment are shows in Fig.2. This is an average value of the evaluation of 144 children speech for each expression words. From this result, there were no expression words with a large mean value, and no expression word which is a feature of children speech was seen. This is thought that the growth of the body affected the voice. Therefore, the average value of the evaluation of expression words was evaluated by age.

\subsection{Change in expression words by age}

Fig. 3 shows the transition of the average value of the evaluation of each expression words by age. From this result, when children age change from 6 years old to 12 years old, we found that children speech changed from high voice to low voice and from unsteady voice to fluent voice. However, other expression words there are not obviously changes founded. So, I conducted a survey by factor analysis.

\subsection{Factor analysis}

Factor analysis ${ }^{(3)}$ was performed using the average value of the evaluation of each expression words with 144 voices. The results are shown in Table 2. Looking at each factor, first of all, classified as factor 1 is breathy voice, noise, non-trembling voice, forceful voice and fluent voice. This is thought to be due to voice quality and how to speak. So I thought that factor 1 is a factor of "Voice quality and how to speak". Next, classification into factor 2 is strength, strained voice and accent. Since this is thought to be due to 
the strength of voice, factor 2 is considered to be a factor of "Voice strength". Factor 3 is classified as high and low, brightness and darkness, clear and resounding. Since this is considered to represent the ease of hearing, factor 3 is a factor of "Ease of hearing". Finally, since factor 4 is an expression word of slow utterance or fast utterance, factor 4 was considered to be a factor of "Voice speed".

\subsection{Difference in feeling between adults and the elderly}

As mentioned above, adults evaluated each phrase in five stages. Therefore, using the evaluation of expressive words constituting each factor, the average value of the evaluation was calculated for each factor. We decided to sort the evaluations of each factor in descending order and use the top $10 \%$ and bottom $10 \%$ speech for the elderly listening experiment. The results are shown in Fig.4. From the Fig.4, there is almost no difference in feeling between adults and older people about Factor 1 "Voice quality and Speaking style" and Factor 3 "Ease of hearing".

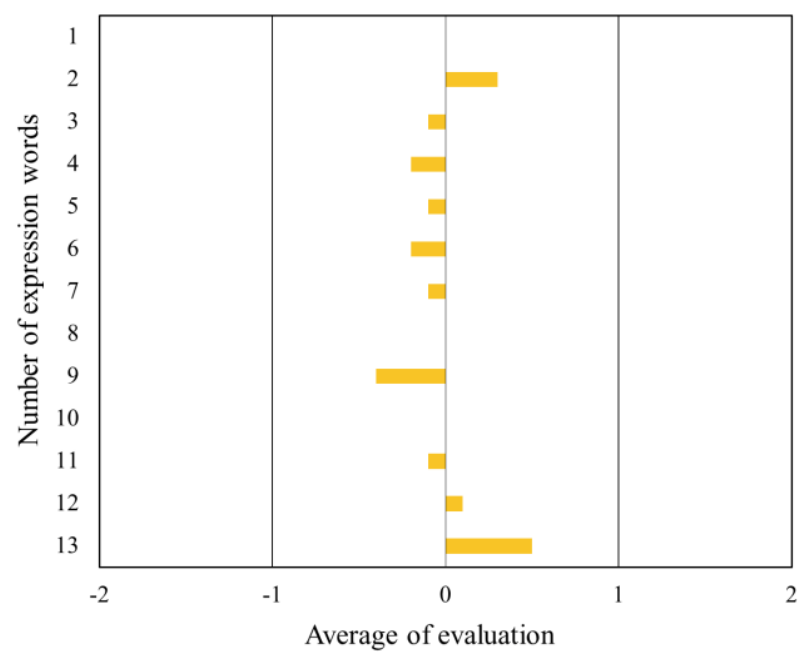

1 Slow utterance / Fast itterance

2 Trembling voice / Non-trembling voice

3 Dark voice / Bring voice

4 Feeble voice / Forceful voice

5 Breathy voice / Non-breathy voice

6 Muffled voice / Modulated and resounding voice

7 Unsteady voice / Fluent voice

8 Speech interspersed with noises from inside the mouth / Speech notinterspersed with noises from inside the mouth

9 Hoarse and rasping voice / Beautiful and clear voice

10 Strained voice / Non-strained voice

11 Low voice / High voice

12 Weak intonation / Strong intonation

13 Weak accent / Strong accent

Fig.2 Evaluation of expression words characterizing children speech

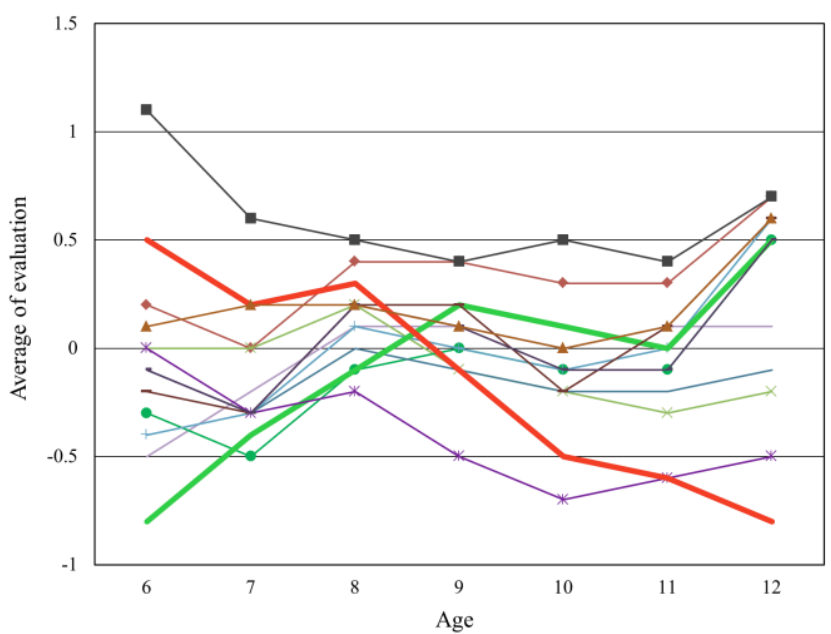

-Slow utterance / Fast itterance

- Trembling voice / Non-trembling voice

-Dark voice / Bring voice

-Feeble voice / Forceful voice

-Breathy voice / Non-breathy voice

-Muffled voice / Modulated and resounding voice

-Unsteady voice / Fluent voice

- Speech interspersed with noises from inside the mouth / Speech notinterspersed with noises from inside the mouth

- Hoarse and rasping voice / Beautiful and clear voice

-Strained voice / Non-strained voice

-Low voice / High voice

$\star$ Weak intonation / Strong intonation

-Weak accent / Strong accent

Fig.3 Change in expression words by age

Table 2 Result of factor analysis

\begin{tabular}{|c|c|}
\hline \multicolumn{2}{|r|}{ Factor 1 "Voice quality and Speaking style" } \\
\hline • & Breathy voice / Non-breathy voice \\
\hline$\bullet$ & Speech interspersed with noises from inside the mouth / Speech \\
\hline & not interspersed with noises from inside the mouth \\
\hline$\bullet$ & Unsteady voice / Fluent voice \\
\hline$\bullet$ & Trembling voice / Non-trembling voice \\
\hline$\bullet$ & Feeble voice / Forceful voice \\
\hline \multicolumn{2}{|r|}{ Factor 2 "Voice strength" } \\
\hline • & Weak accent / Strong voice \\
\hline • & Strained voice / Non-strained voice \\
\hline • & Weak accent / Strong accent \\
\hline \multicolumn{2}{|r|}{ Factor 3 "Ease of hearing" } \\
\hline • & Low voice / High voice \\
\hline$\bullet$ & Hoarse and rasping voice / Beautiful and clear voice \\
\hline$\bullet$ & Dark voice / Bring voice \\
\hline$\bullet$ & Muffled voice / Modulated and resounding voice \\
\hline \multicolumn{2}{|r|}{ Factor 4 "Voice speed" } \\
\hline$\bullet$ & Slow utterance / Fast utterance \\
\hline
\end{tabular}




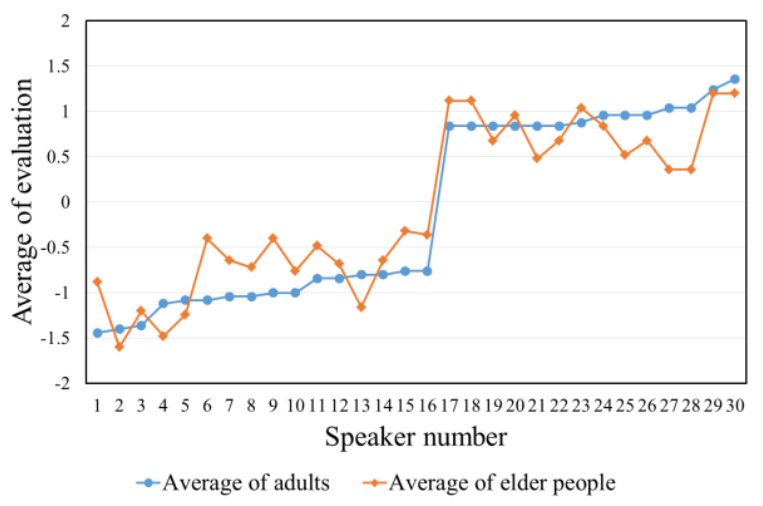

(a) Factor 1

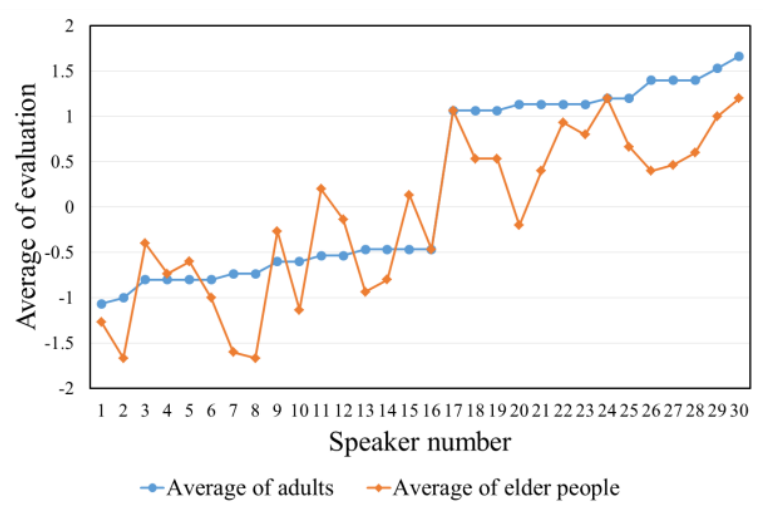

(b) Factor 2

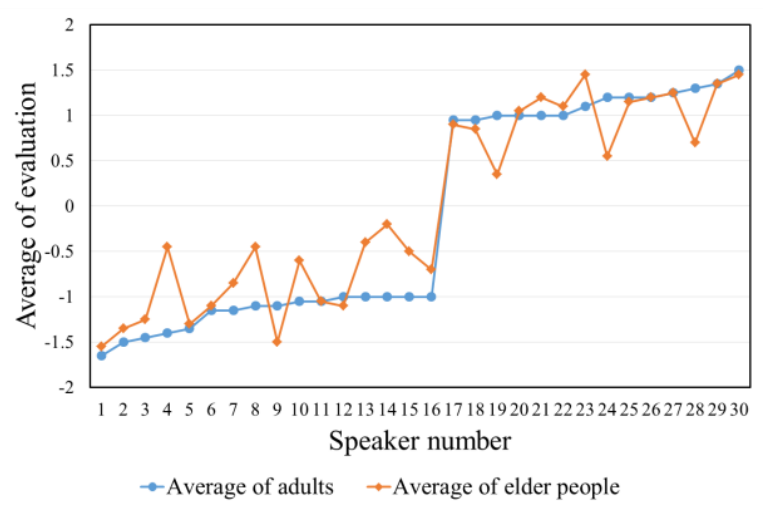

(c) Factor 3

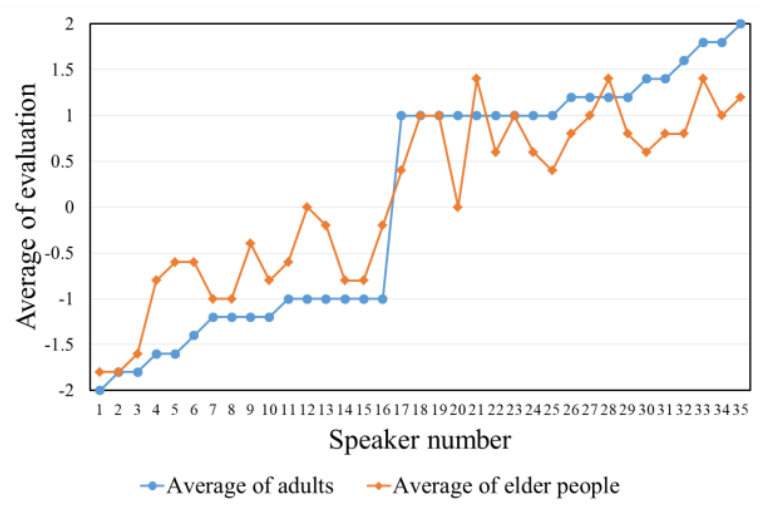

(d) Factor 4

Fig.4 Difference in feeling between adults and the elderly
However, Factor 2 "Voice strength" is not strongly felt by elderly people as adults feel strong. Also, Factor 4 "Voice speed" is where both the adult and elderly feel slow, but the elderly feels not so obviously.

\section{Conclusions}

In this research, as a preliminary stage of processing for hearing aids users to hear the children speech easier, feature of children voice using expression words were examined. As a result, when children age change from 6 years old to 12 years old, children speech changed from high voice to low voice and from unsteady voice to fluent voice. In addition, expression words that characterize children speech are classified into four factors of "Voice quality and how to speak", "Voice strength", "Ease of hearing" and "Voice speed" in this study. The difference between how adults and elderly feel the children speech is: For factor 1 and factor 3, the adults and elderly gave an almost same evaluation result; For factor 2, the elderly feel less strong when adults are feel strong on "Voice strength"; And for Factor 4 the adult feels slow at "Voice speed", but the elderly people did not feel so slow. In the future, we are planning to applicate this result to examine if it makes the hearing aid user hear the children speech easily.

\section{Acknowledgment}

Authors sincerely appreciate the National Institute of Informatics for their warmly support and offer the speech database.

\section{References}

(1) “JapanTrak2015”, 60-62, 2015

(2) Takeshi Miyazaki : "Selection of Japanese adjectives for characterizing elderly speech", Spring Meeting Acoustical Society of Japan, 2008

(3) Hiroshi Shimizu : "An introduction to the statistical free software HAD: Suggestions to improve teaching, learning and practice data analysis", Journal of Media, Information and Communication, 1, 59-73, 2016 